\title{
Active Magnetic Suspension and Bearing
}

\author{
Adam Krzysztof Piłat \\ AGH University of Science and Technology \\ Poland
}

\section{Chapter overview}

The aim of this chapter is to present investigation, modelling and simulation of Active Magnetic Levitation Systems (AMLS). Active means that the operating mode is fully controlled with desired performance specified by a user defined controller law. Active Magnetic Suspension (AMS) and Active Magnetic Bearing (AMB) are AMLS representatives. These systems can be characterized as the mechatronics devices because they combine a number of technologies: mechanics, electrical engineering, electronics, computer science and control theory. These devices are structurally unstable and the application of a control law is necessary for the stable operation in levitation mode. To levitate an object the appropriate value of electromagnetic force has to be generated by the stabilizing controller. In the AMS the electromagnetic force counteracts the gravity force to obtain object levitation while in the AMB the electromagnetic forces counteract to each other and to gravity. The electromagnetic force is generated by the electromagnet consisting of the stator of a specific design as far as the shape and number coils are concerned. The electromagnets are formed in the $\mathrm{C}$ or E shapes typically. One of advanced modelling and designing tasks is to find the optimal shape for the particular solution. At the modelling stage the graphical form of the electromagnet can be represented by a set of graphical primitives. Using Bézier curves it is possible to obtain the parameterised and continuous electromagnet shape which could be optimised under many criteria: maximal force, Eddy current losses, resistive heating, flux saturation, controller quality, required stiffness and damping. All these problems ought to be analysed at the modelling, simulation and experimental investigation stage.

Typically AMLS models are based on ordinary differential equation parameterised by the optimisation of identification results. The nonlinear form of these equations allows to describe almost all phenomena existing in the particular construction and configuration of power actuators, sensors and control system. The experimental investigation is required for better understanding of a problem and for model verification but construction aspects, used materials are still ambiguous, thus it is very difficult to obtain a general model to be used in the future. Better is to say that the knowledge base has been achieved that describes a particular AMLS application. Nowadays control and construction designers are aiming at the ideal system similar to ideal set of springs and dampers working in the whole operating range with programmable stiffness and damping. Thus, at the modelling stage the construction properties and controller design ought to be analysed in a complex form. The controller is designed when the working system was investigated and 
modelled. In this case the controller must be fitted accurately for the required application performance.

The author recommends to include the control strategy into the designing phase. AMLS are specific mechatronics devices in which the construction and control laws are strongly coupled. The modern COMSOL Multiphysics (Comsol, 2007) software allows to perform multidisciplinary analysis based on partial (PDE) and ordinary (ODE) differential equations, using material and geometry properties. The controlled magnetic field is studied under the satisfying geometry and current flow constraints. Due to the COMSOL Multiphysics software the interdisciplinary model can be obtained and embedded into MATLAB/Simulink for the closed loop simulation purposes. This feature has newer been studied in the literature despite that research and it gives an unexpected possibilities in the AMLS optimal design and control.

\section{Introduction}

The Samuel Earnshaw theorem (Earnshaw, 1842) proves that it is not possible to achieve a static levitation using any combination of fixed magnets and electric charges. The static levitation means the stable suspension of an object against gravity. However, there are a few exceptions around it which violate the assumptions (Braunbeck, 1939), (Jayawant, 1981): quantum mechanics, rotation, diamagnetism, oscillating fields and feedback. This chapter deals with feedback systems. The first recorded system which offered an alternative to the wheel-on-rail was the floating train developed by Girard in 1864 (Sinha, 1987). In 1937 Beams and Holes used an electromagnetic suspension for testing material strength. Their patent related to AMB is dated to 1941. The AMB technology started to operate with digital control technology under work of Habermann (Habermann \& Liard, 1979) and Schweitzer (Schweitzer, 1976). The first international symposium on the active magnetic bearing technology was held in 1988 with the founding of the International Society of Magnetic Bearings by Prof. Schweitzer, Prof. Allaire (University of Virginia), and Prof. Okada (Ibaraki University).

Nowadays, worldwide there is a number of research centres and companies focused on the AMS and AMB research. They have their own individual test-rigs and particular experience in this research domain. The results are presented and discussed by researchers and development engineers during the International Symposium on the Magnetic Bearings (ISMB) event that is organized once every two years. Another conference that shares the issue of the magnetic levitation technology is the International Conference on Magnetically Levitated Systems and Linear Drives. The research of the AMS properties and control allows to transfer knowledge and methods to the $\mathrm{AMB}$, which is a part of it. The advances in material, microelectronics, computer science, nonlinear control, modelling and simulation have made the AMLS a more available technology up to now. Nowadays, a detailed analysis and optimisation of all the AMLS components are required to minimize its complexity and high costs.

In recent years a number of machines with Active Magnetic Bearings have been designed in order to eliminate the lubricant medium, vibration, noise and to achieve high velocities and loads. These systems are complicated due to mechanical, electrical and electronic circuits construction. A wide range of analyses is required during the designing procedure - from the construction stage up to the development of the control algorithm architecture. The finite element method can be a tool for the magnetic field analysis. Many scientists working 
on magnetic bearings or self bearing motors use this method at the designing stage. The numerical analysis allows to check if the new proposed AMB structure is effective with respect to levitation forces, magnetic field properties and electro-mechanical interactions.

The finite element method was also used to analyse the air gap flux and radial forces in the miniature self bearing motors (Kanebako at al., 2002), (Ohmori at al., 2002). In small-sized systems essential modelling errors are often caused by leakage and nonlinear effects that could be neglected in larger systems. The appropriate AMB construction is a trade-off among many requirements and should be specific for different applications. The development stage of a machine equipped with the AMB demands co-operation of many experts in mechanics, structure of materials, electronics and control. The proper machine construction, optimal AMB structure and dedicated control algorithm allow to achieve a modern industrial unit.

The basis of the AMLS operation is an electromagnetic force generated by the electromagnet (see Fig. 1) that can be calculated using Maxwell's equations.

a)

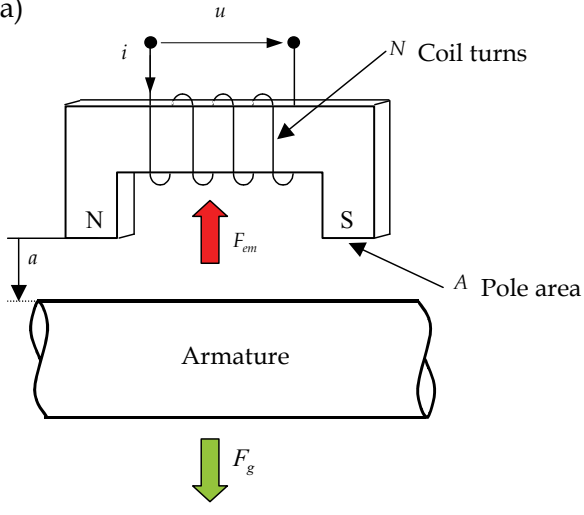

b)

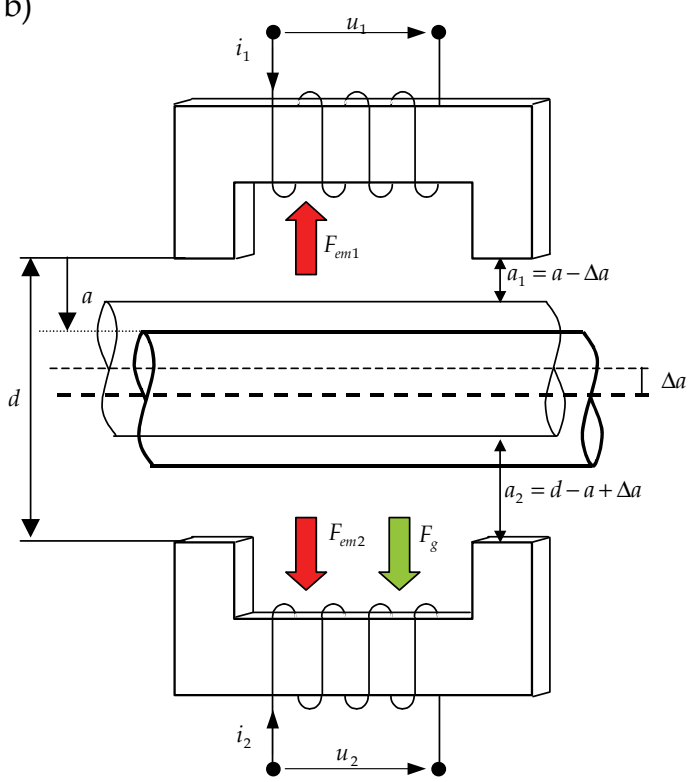

Figure 1. Armature suspension in two configurations: a) single electromagnet, b) two electromagnets

It is a set of equations, describing relationships between the fundamental electromagnetic quantities. These quantities are the electric field intensity $E$, the electric displacement or electric flux density $D$, the magnetic field intensity $H$, the magnetic flux density $B$, the current density $J$, and the electric charge density $\rho$. For general time-varying fields, Maxwell's equations and equation of continuity can be written as differential equations that the finite element method can handle. 


$$
\begin{gathered}
\nabla \times H=J+\frac{\partial D}{\partial t}, \nabla \times E=-\frac{\partial B}{\partial t} \\
\nabla \cdot D=\rho, \nabla \cdot B=0 \\
\nabla \cdot J=-\frac{\partial \rho}{d t}
\end{gathered}
$$

The electromagnetic force is calculated using magnetic field energy $\mathrm{W}$ :

$$
F_{e m}=-\frac{\partial W}{\partial a}, W=\frac{1}{2} \int B H d V
$$

where $F_{e m}$ is the electromagnetic force, $a$ is the distance between field source and armature and $V$ is the air gap volume. Setting the armature at desired distance $a$ the electromagnetic force can be calculated with respect to the flux density, coil current $i$, coil inductance $L$ and armature distance $a$.

$$
F_{e m}(B)=A \cdot B^{2} \mu_{0}^{-1}, F_{e m}(a, i)=-\frac{K}{4} \frac{i^{2}}{a^{2}}, F_{e m}(a, i)=\frac{1}{2} \frac{d L(a)}{d a} i^{2}
$$

where: $K=\mu_{0} N^{2} A$ is an electromagnet constant, $A$ is a pole area, $\mu_{0}$ - permeability of vacuum and $N$ - no of coil turns.

\section{Principles of active control}

As mentioned before the controller is required to obtain the stable levitation with the desired performance of the suspended object. The complex controller has to realize many tasks (Maslen, 1995), (Schweitzer, 1994): coordinate transformation of sensor signals, collection of any other parameters needed by control algorithm, generation of control current (or flux) requests: executing control algorithm, coordinates transformation, biasing of amplifier signals and additionally permit modification of control algorithm, implement diagnostic measurements.

The controller can be realized in the analog or digital form. They serve worse or better to the control goals and both of them have some advantages and disadvantages. The analog controller is based on op-amps, resistors and capacitors and generally performs a linear control strategy. It is an inexpensive and simple solution but has a fixed configuration and a component drift can occur. The digital control technology is based on FPGAs, DSPs and microcontrollers together with $\mathrm{A} / \mathrm{D}$ and $\mathrm{D} / \mathrm{A}$ converters. The controller complexity is high but linear and nonlinear control strategies can be easily implemented and modified (Grega \& Piłat, 2001). With-this technology some additional control, diagnostic and monitoring features can be implemented.

The ferromagnetic suspension system is inherently unstable and thus the active control needs to be incorporated for stable suspension. The basic principle of the AMLS operation is to apply the voltage to an electro-magnet to keep a ferromagnetic armature levitated. The function of a control action is modifying the force-distance characteristics to obtain required 
stiffness and damping properties. The control can be realized in the explicit feedback (the external sensor is used to measure the distance or flux) or the implicit feedback (based on changes of the magnet coil inductance owing to the variations in the air gap). Additionally, the coil current is measured to explore identification and multi loop or nonlinear control strategies.

One of electromagnets of the AMLS can be analysed as the single-degree-of-freedom massspring-damper system with controllable stiffness and damping. Using the non-contact actuator both parameters are controlled by the formulated control strategy and when choosing the appropriate values of the poles we can obtain the required dynamic behaviour of the closed loop. The controller parameters can be designed to satisfy requirements of the closed loop performance determined by the undamped natural frequency and the damping ratio. Choosing the appropriate values we can control the speed of the system response to the external disturbance. The highest natural angular frequency gives faster system response and the damping mode can be controlled after setting the appropriate value of damping ratio.

a)

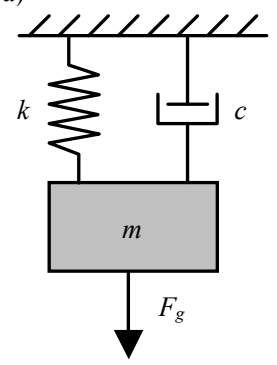

b)

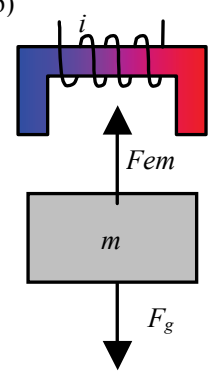

c)

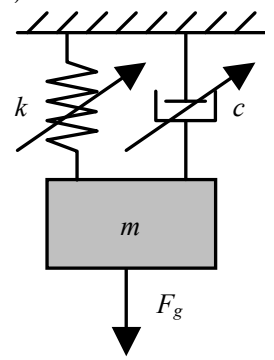

d)

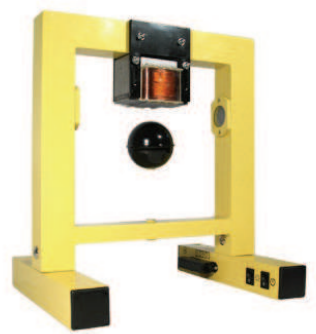

Figure 2. Object suspension: a) a typical mechanical approach - the spring mass-damper system, b) electromagnetic suspension, $c$ ) non-contact system equivalent to the controllable stiffness and damping, d) MLS0201 - active suspension at work (Piłat, 2005a)

Mounting the second electromagnet opposite to the first one we can obtain the differential model of the controlled armature (eq. 5). Controlling the damping and stiffness in the course of action both electromagnets ought to be controlled independently or in a differential mode. In this case the gravity factor could be neglected if the electromagnetic forces are much higher than the gravity one. In this case much higher stiffness and damping can be obtained. This kind of configuration is the basic solution in the typical AMBs, where four electromagnets are used. The control law can be applied separately to each of them, to both pairs or in a global case to all of them.

$$
\begin{gathered}
m \ddot{a}=F_{e m}+F_{g} \\
m \ddot{a}=F_{e m 2}-F_{e m 1}+F_{g}
\end{gathered}
$$


a)

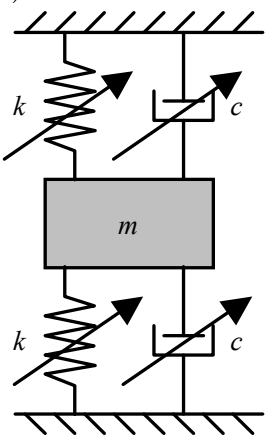

b)

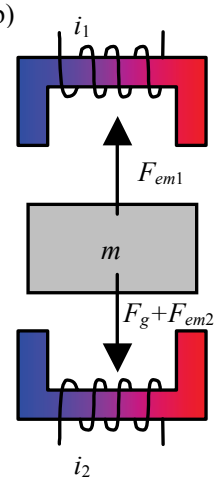

c)

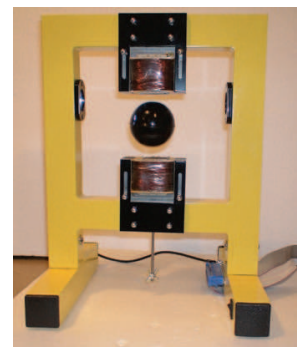

d)

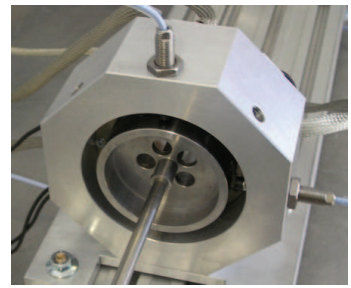

Figure 3. Electromagnet counteraction: a) mechanical representative, b) non-contact system equivalent to the controllable stiffness and damping, b) MLS0202 - laboratory test-rig, c) radial AMB (Piłat 2006)

Another possibility to simplify the AMB construction is to use three poles only (Meeker \& Maslen, 2006) or to eliminate one of electromagnets. Three sources of the magnetic field are a necessary minimal number of actuators required for the rotor levitation in the bearing space. A small number of execution units and electronics circuits allow to minimize a bearing size, production costs and energy consumption. The disadvantages of this solution correspond to the slighter stability region and strong nonlinearities. The rotor suspension can be described by equation (6) (Gosiewski 1993, Kozanecka 2000).

a)

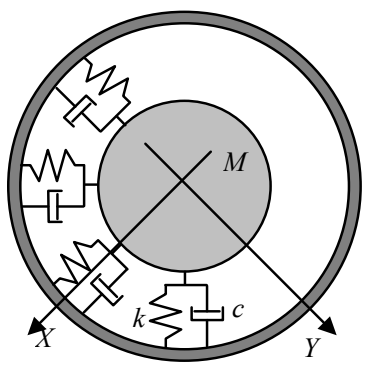

b)

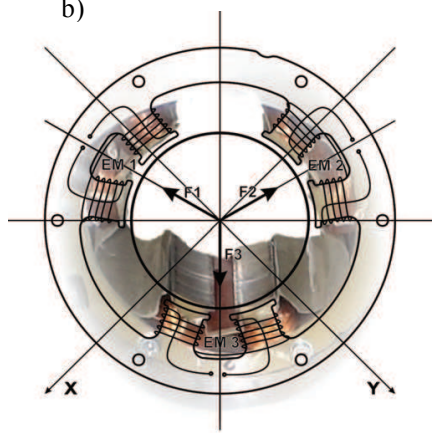

c)

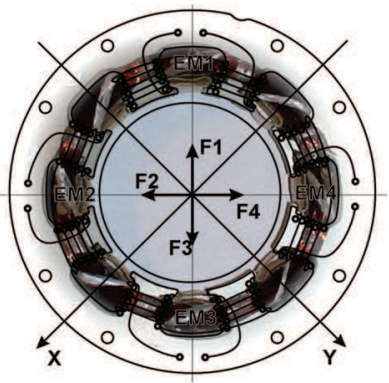

Figure 4. Three and four electromagnet based AMB constructions: a) general model, b and c) author's AMB prototypes

$$
M \ddot{q}+C \dot{q}+K q=F
$$

Where $q$ is a state vector, $F$ is vector of forces and $M, C, K$ are mass, damping and stiffness matrices respectively.

The nonlinear and robust control strategies are studied to maximize the performance of the AMLS. The power of automatics and control theory implemented in digital or analogue programmable device (Piłat, 2005a) allows to reconfigure controller configuration while operating. 


\section{Mechatronics approach}

The Active Magnetic Suspension and Active Magnetic Bearings can be categorized as mechatronics systems. Mechatronics is the synergistic combination of precision mechanical engineering, electronic control and systems thinking in the design of products and manufacturing processes. It relates to the design of systems, devices and products aimed at achieving an optimal balance between basic mechanical structure and its overall control.

The new technology devices equipped with electromagnetic actuators require precise design and manufacturing. The AMLS consist of components from a few brands (techniques) mechanics, electrical engineering, electronics informatics and automatics). The typical and mostly used designing path is based on a few steps realized in the serial way: calculation stage, mechanical construction design, sensors, actuators and control units design, integration and application of control strategy. Such a designed and manufactured prototype is identified and tested practically. After collecting the identification results and handling the data the system knowledge base increases and can be used to modify the existing project components and in a project development. Every project modification requires to rebuild a prototype, which could be an expensive task. On the other hand, this knowledge is still limited to the prototyped devices family. The prototyping path could include identical or similar devices.

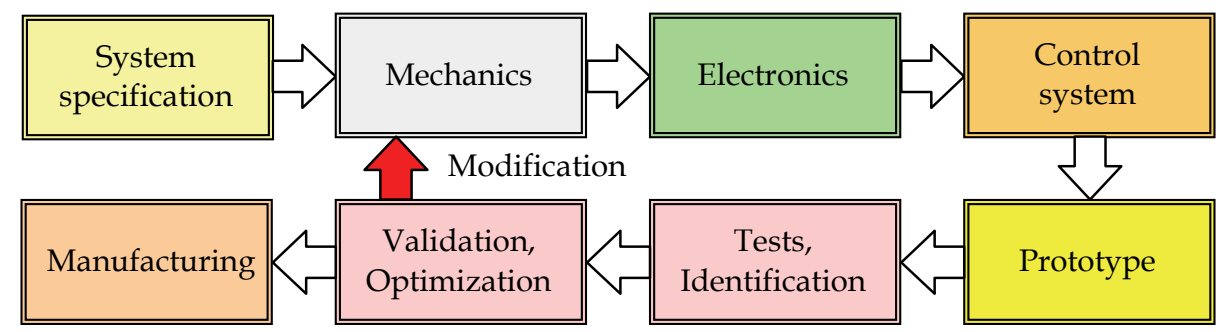

Figure 5. Traditional Design Approach - particular elements are designed separately and then the prototype is mounted and analysed. Each of the prototyping stages is well modelled and simulated, but does it satisfy the perfect prototype operation?

Moreover, the AMLS operation is based on the control strategy that is customized to the system application and it should be done in an optimal way to satisfy the application requirements. Thus, the conventional modelling, simulation and prototyping path is insufficient for levitation systems. So it is necessary to develop a method for modelling and simulation of a complex levitated system. The research being realized with typical and well known CAD/CAM tools, MATLAB/Simulink calculation package and custom hardware has shown that it is desirable to perform complex modelling and simulation of the AMLS. This kind of analysis consists of the actuator unit properties including materials and construction characteristics and complies to physical phenomena related to the system operation. Thus, the virtual prototype of AMLS is proposed. This prototype should be designed in a parallel way with an interdisciplinary analysis feature. The development of such complex model will enable effective and optimal designing of the electromagnetic actuator together with a control strategy. 


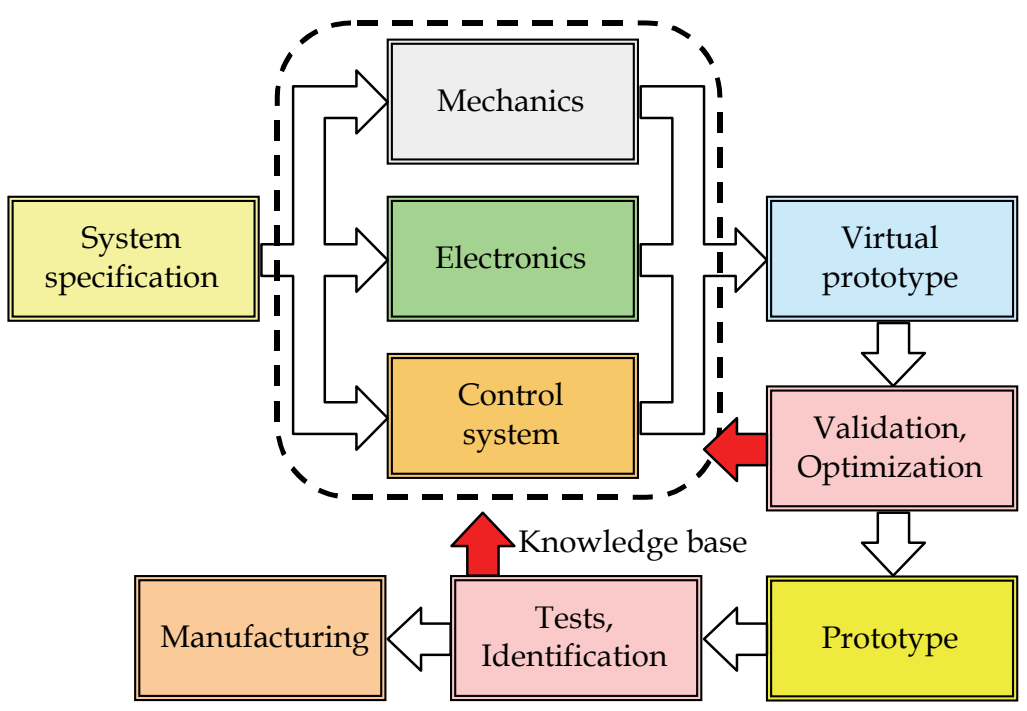

Figure 6. The proposed interdisciplinary Design Approach where virtual prototype is being developed and studied

Nowadays, most of the automatics execution units and mechatronic systems, and particularly actuators for levitated systems are designed and tested in the integrated MATLAB/Simulink package. A set of analytical calculations is performed, where the modelling of systems dynamics is studied using ordinary differential equations. The dynamics is formulated on mathematical models under a set of assumptions that simplifies formulas and dependencies, but can limit the real-system behaviour. To extend traditional modelling and simulation stage an interdisciplinary approach is proposed.

\section{Modelling and simulation}

In the realized research the analytical approach is being used to solve differential equations and calculate surfaces and volumes. These calculations are supported by numerical computing. The static and dynamic properties of the AMLS are modelled and simulated by ordinary differential equations in MATLAB/Simulink environment, which is nowadays the most popular and advanced engineering and scientific tool. A wide range of toolboxes especially dedicated to control and optimisation problems allows to design and perform specialized analysis of the considered control problem. The proposed control strategies are also implemented and tested in MATLAB/Simulink package. The realized models contain system specifics characterized by its architecture, sampling frequencies, used devices (e.g. $\mathrm{A} / \mathrm{D}$ and D/A quantization effects), serial and/or parallel data processing. The real-time environment and dedicated boards with user-defined signal processing (Piłat 2002a, Piłat 2006) are used to collect the data for model verification purposes. The investigation of 
AMLS is realized with the parallel signal data acquisition that cancels any delays and guaranties causality of component behaviour.

During the design of AMS (Piłat, 2003), (Piłat \& Turnau, 2005) and the reconfigurable laboratory test-rig for the AMB (Piłat, 2004b), (Piłat \& Grega, 2005c) it has been noted that a complex interdisciplinary analysis is necessary to achieve an almost real model of such systems. The constructed prototype gives a number of information about system static and dynamic properties. Applied sensors allows to identify existing physical phenomena's and their cross couplings. The designed AMLS prototypes have been designed before FEM tools were used at the modelling and simulation stage. The analysis of the existing constructions and collection of identified data build the knowledge base and is used at the FEM modelling and simulation verification stage.

COMSOL Multiphysics (COMSOL, 2007) is a powerful interactive environment for modelling and solving all kinds of scientific and engineering problems based on partial differential equations (PDEs). To solve the PDEs, COMSOL Multiphysics uses the proven finite element method (FEM). The software runs the finite element analysis together with adaptive meshing and error control using a variety of numerical solvers. A user can perform various types of analysis including stationary and time-dependent analysis, linear and nonlinear analysis, eigenfrequency and modal analysis. The user can operate a well designed graphical interface and/or COMSOL Script. Using a script programming method the user can apply data structures and functions to realize non-standard or multidisciplinary modelling. This feature allows to customize designing and analysis procedures. The full interface to MATLAB/Simulink environment allows to realize interdisciplinary analysis, which is very important from the automatic control point of view. When using FEM for AMLS it is important to accurately select mesh properties due to a bigger difference between device sizes and the levitation area (e.g. 50000:1), thus, after shape discretization the quality of the generated mesh should be measured and analysed. This feature is offered by the COMSOL Multiphysics software where the user can set predefined or individual settings for the selected regions and mesh them with required accuracy. Taking into consideration the levitated systems, the modelling of the air gap with the specified accuracy is crucial for electromagnetic force and system dynamics analysis, but strongly increases computational effort.

The electromagnetic force generated by the electromagnet depends on its geometry, coil parameters, coil current and ferromagnetic object distance from its front side. Nowadays, all these parameters play an important role in the efficient and optimal electromagnet design for magnetic suspension and active magnetic bearings applications.

The application of the magnetic field for an attraction of ferromagnetic objects requires proper electromagnet construction design and plays an important role in the control applications.

The magnetic properties of the used materials can be entered and used in the modelling process. The same can be done with coil parameters, current density and distance. Such a prepared model represents an ideal form of a modelled system and ought to be verified.

Since many years the author has been interested in a complex system modelling and simulation. Nowadays it becomes available. The only limits are introduced by a quasi realtime computer operation. For the control research it would be nice to have an adequate model of the real system. The designed set of identification tools allows to model the 
dynamics of the AMLS and design control strategy which is verified on the laboratory testrigs.

The most important problem is to have a realistic model of the AMLS. As mentioned before the AMS and AMB are complex mechatronics systems and they should be designed under consideration of all possible physics phenomena and particularly under the controller supervision. While developing new constructions and control strategies the researcher ought to think in an interdisciplinary way to fulfill the analysis of interaction between all physical fields existing in the levitated system under active control. Three steps in modelling and simulation: geometry modelling, magnetic field analysis and dynamics simulation in an interdisciplinary environment are presented in the consecutive points.

\subsection{Modelling actuator geometry}

When analysing the equations (3) one can notice that the electromagnetic force depends on the medium volume existing between the levitating object and electromagnet. The assumption of the parallel armature position to the electromagnet is mostly used when the force is calculated and therefore, the model operates well for small distances and regular shapes. Generally, the levitated object has a different shape e.g. spherical or cylindrical and the electromagnet poles can be located at the circumference. In the most general form the air gap volume and its reluctance ought to be included in the model. As mentioned above the electromagnet and object geometries are important while the magnetic field is formed to interact between each other. The geometry of a particular object is designed with the CAD software support. Most of them is based on geometry primitives - lines and arcs created with the use of the manual drawing procedure and/or Boolean operations.

The first approach to the AMLS interdisciplinary modelling was based on the designed CAD drawings. The exported stator shapes were used at the modelling stage to carry magnetic flux. The COMSOL CAD translation feature allows to save a great deal of time when laying out large or complex models. The designed electromagnet shapes can be imported from DXF files and then used at the magnetic field modelling stage. The second method based on graphical primitives and Boolean operation allows to simply create an electromagnet model corresponding to the standard laminations. The recommended method for creating a virtual prototype in an automatic way is based on the programming approach using the analytical form of polylines including Bézier curves (Piłat, 2007). The obtained form of the AMB components (see Fig. 7) can be easily used at the optimisation stage to find an adequate form of a bearing to a particular application. This modelling tool allows to parameterise the whole AMLS geometry along with rounded corners.

The AMB front and back planes are parallel, thus the 3D form can be extruded from the $2 \mathrm{D}$ shape. In this case the modelling process can be performed in the 3D space without extra CAD/CAM software interaction. Moreover, the drawing resolution set in 2D is kept in $3 \mathrm{D}$ space. Figure $7 \mathrm{c}$ presents a $3 \mathrm{D}$ view of the AMB prototype form with a specified depth. 

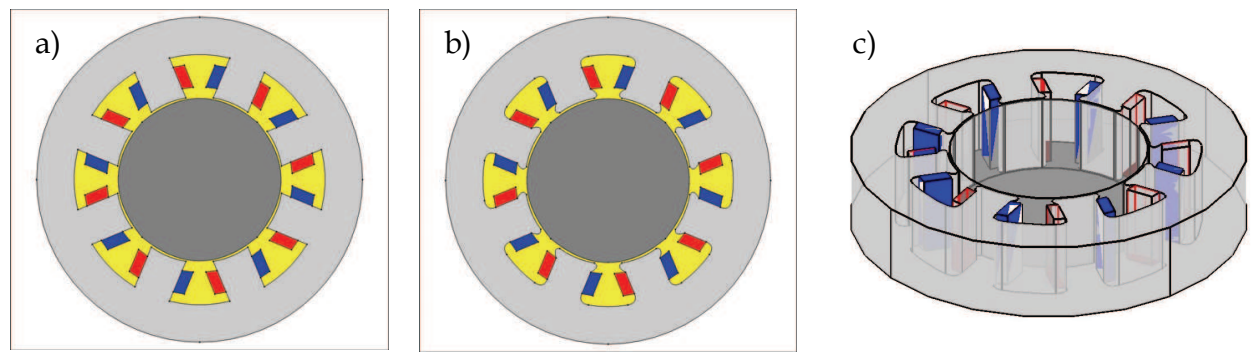

Figure 7. Automatically generated AMB shapes: a) standard form b) smooth form c) 3D model

The same methods can be applied to any electromagnet design and/or electrical drives. The geometry components account for an initial form for the interdisciplinary modelling stage.

\subsection{Modelling magnetic field}

The results presented in this chapter have been obtained using the magnetostatic mode for analysis purposes. The magnetostatic module is one of the COMSOL software features based on Maxwell's equations.

Four areas can be considered in the analysed example (Fig. 8a):

- Two ferromagnetic elements: the stator in three parts and the shaft. The stator core consists of thin laminated electrical steel plates for the purpose of reducing eddy current effects and characterized by a nonlinear function of relative permeability. The shaft is made of iron block.

- The air gap that fills the area between the stator, rotor, coils and the shaft is assumed as a paramagnetic region.

- Coils made of copper wire wound up on the stator pole shoe. The magnetizing current is required to set up a given magnetic flux in the iron circuit. The number of turns and the current level determine the flux intensity and the electromagnetic force, respectively. The coil along with the pole shoe make an electromagnet called the solenoid actuator.

a)

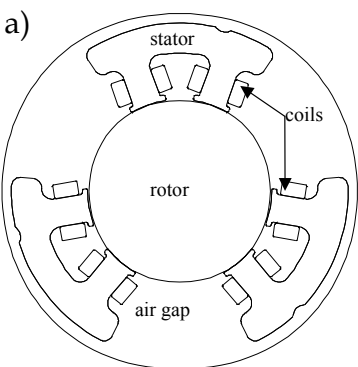

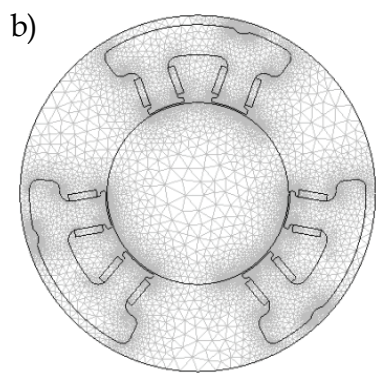

c)

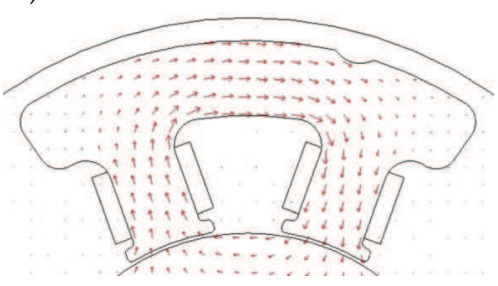

Figure 8. AMB FEM modelling a) subdomains, b) mesh, c) magnetic flux density in the selected stator 
The results of the previous research (Piłat, 2004) have been very useful in the three-coil AMB design. The experience in the CAD design and the import advantages of the COMSOL CAD files have been very helpful in the model formulation (Fig. 8a). Coils represented by the rectangular model components have been formulated in the COMSOL Draw Mode to achieve the required quality of the adherent regions. For the precise CAD import it is recommended to select import parameters with respect to the geometric sizes. the appropriate material qualities have been assigned to the selected subdomains. The coil current density has been set using parameters for easy value modification. In this study 2 rotors have been considered: the pipe-type and the solid ones. The next step in the analysis of the bearing construction is the mesh generation (see Fig. 8b). The mesh parameters have been set to achieve the best performance in the most important regions: the interior of the stator and the air gap between the rotor and stator.

For the desired coil parameters: a number of turns, the current value and the coil intersection surface it is possible to calculate the current density. The nonlinear function of electrical steel magnetization (see Fig. 12b) has been used in sequence to obtain realistic results of calculations. The performed analysis of the magnetic flux flow through the magnetic core shows that the areas with the highest flux density are concentrated at the stator edges and coil windings. Smooth edges in the stator construction result in the minimization of the flux concentration. The highest density of the magnetic flux is located in the stator pole shoes at the shortest path of the magnetic flux. The flux direction flow is determined by the coil current direction (Fig. 8c). The analysis of the rotor influence shows that it is possible to modify its construction while the parameters of the magnetic circuit remain the same (Fig. 9).

a)

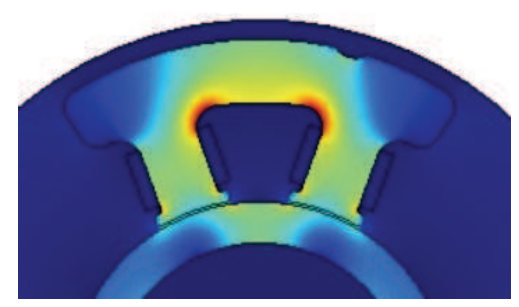

b)

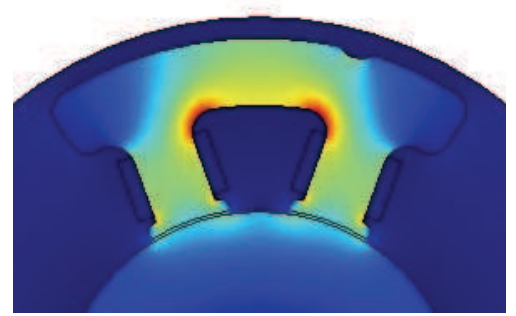

Figure 9. Magnetic flux density in the selected stator with two types of the rotor: a) pipetype, b) disk-type

The research that follows focuses on the shaft movement influence on the magnetic field in the AMB. Figure 10 presents the potential of the magnetic field while the rotor is located at the bearing centre and moved vertically 300 micrometers up and down with respect to the bearing centre. The potential of the magnetic field is changed due to the rotor motion while the current density remains the same. This fact is obvious but important for the AMB dynamics modelling and the controller synthesis. In case of the rotor movement the air gap width changes under the pole shoes. The air gap change is nonlinear due to the circular shaft shape and the air gap size with respect to the shaft diameter. In case of the three-coil AMB this nonlinear gap change plays an important role in the nonlinear bearing dynamics modelling and the controller synthesis. The magnetic flux change strongly depends on the distance between the shaft and the pole shoe. Thus, when the air gap decreases, the magnetic flux increases, and conversely (Fig. 10). 
a)

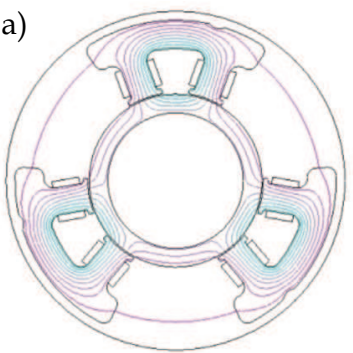

d)

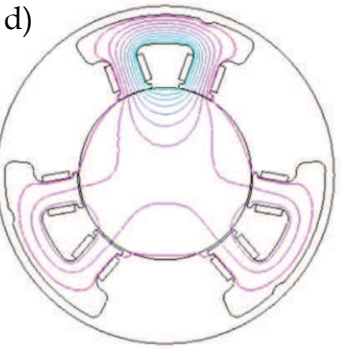

b)

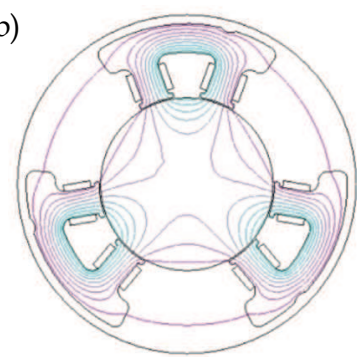

e)

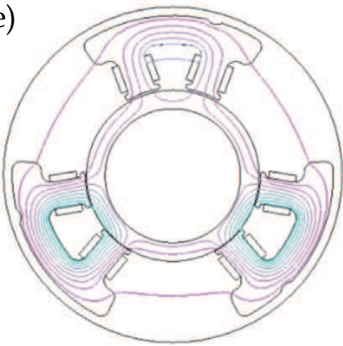

c)

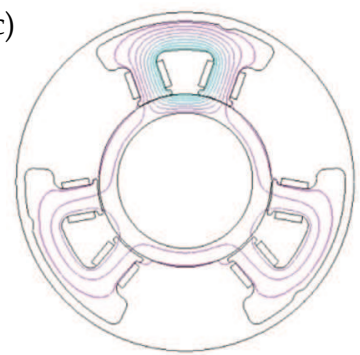

f)

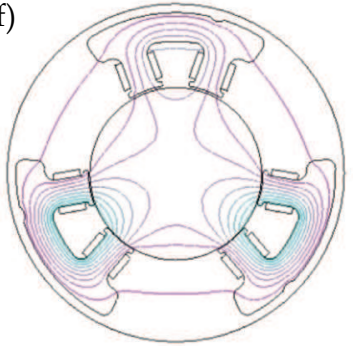

Figure 10. Magnetic potential in the AMB at various rotor configurations

In conventional active magnetic bearings the rotor is stabilized by the control algorithm based on the rotor displacement measurements. High quality eddy currents probes are used to precisely measure the rotor position in the bearing area. The aim of the research that follows is to analyse the flux sensor application. That part of the bearing stator has been specially designed (see Fig. 11a). While using the COMSOL feature that allows to measure field properties at the defined point the magnetic potential was measured at different rotor displacements. For the selected electromagnet three additional points (Left, Sensor, Right) were created for postprocessing purposes (see Fig. 11a). In the course of the application of the selected points the magnetic field potential $(\mathrm{Wb} / \mathrm{m})$ has been evaluated.
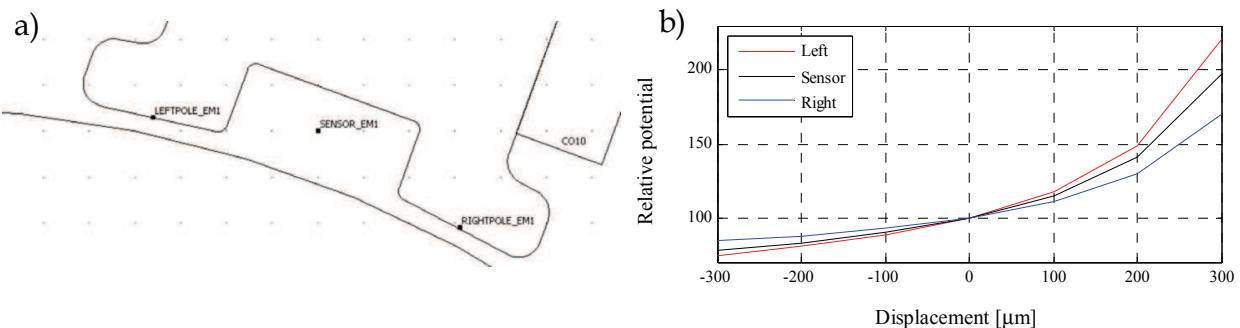

Figure 11. The designed pole for sensing purposes with diagnostic points (Left, Sensor, Right) and simulated relative potential

The magnetic field calculation procedure consists of the following steps: modification of the rotor location at the bearing plane, solution of the problem, data reading form the specified points. This procedure has been executed for the vertical and horizontal rotor movement. The obtained magnetic potential values were related to the value calculated for the central 
rotor position (Fig. 11b). This factor shows changes in the magnetic field when the rotor movement occurs.

The vertical movement represents the axial movement regarding the upper stator axis. Positive values correspond to the rotor movement in the upright directions. The horizontal rotor movement is realized in the perpendicular axis and positive values indicate the right side location. This analysis shows that the sensor can be located in the stator pole. Its displacement characteristics are nonlinear and depend on the rotor movement. For the rotor variations up to $10 \%$ of the nominal radial air gap the sensor characteristics can be successfully linearized which is favourite for the controller design and operation.

\subsection{The AMLS interdisciplinary modelling and simulation}

The presented magnetostaic approach is one of the modes that are available in COMSOL Multiphysics. Considering the AMLS the dynamics study is the most important condition of modelling and simulation. The formula (4) describing the levitated object dynamics contains the electromagnetic force which ought to be calculated with the accurate configuration of the device. With Maxwell equations this force could be calculated as the surface stress tensor. The following appropriate qualities are set to the AMLS subdomains: copper, air, iron, magnetisation curve for stator steel material (See Fig. 12a). The physical properties of electrical steel are measured (at $50 \mathrm{~Hz}$ ) and specified by manufacturers (see Fig. 12b).
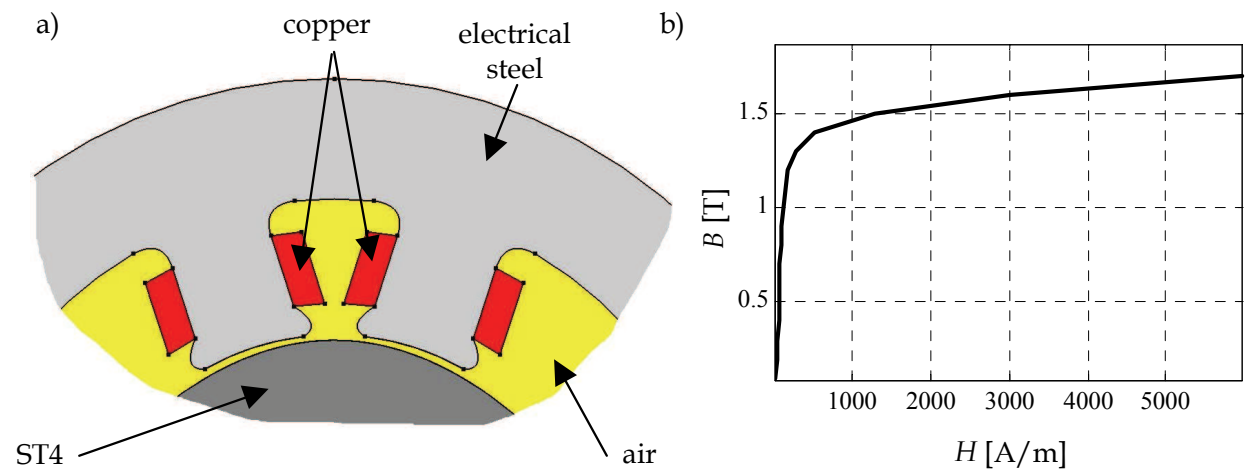

Figure 12. Materials assignment: a) AMB subdomains, b) nonlinear magnetization curve of non oriented steel strip M330-50A. (On courtesy of STALPRODUKT-Bochnia S.A.)

The realistic model of the system is obtained. The appropriate PDE related to the AC/DC perpendicular currents module allows to calculate the electromagnetic field affecting the object. The dynamical movement is described by the ODE equation and the state variables are used to modify the moving mesh status. With this feature the dynamical behaviour of the levitated object can be studied. Moreover, the coil current is controlled from the MATLAB/Simulink where the appropriate controller is implemented. Taking this into account, all existing models and designed controllers can be used in this complex multiphysics modelling and simulation mode. Moreover, the created interdisciplinary model can be extended with additional modes: e.g. heating, fluid. Having a set of PDEs the complex multiphysics model representing the real system can be achieved. 


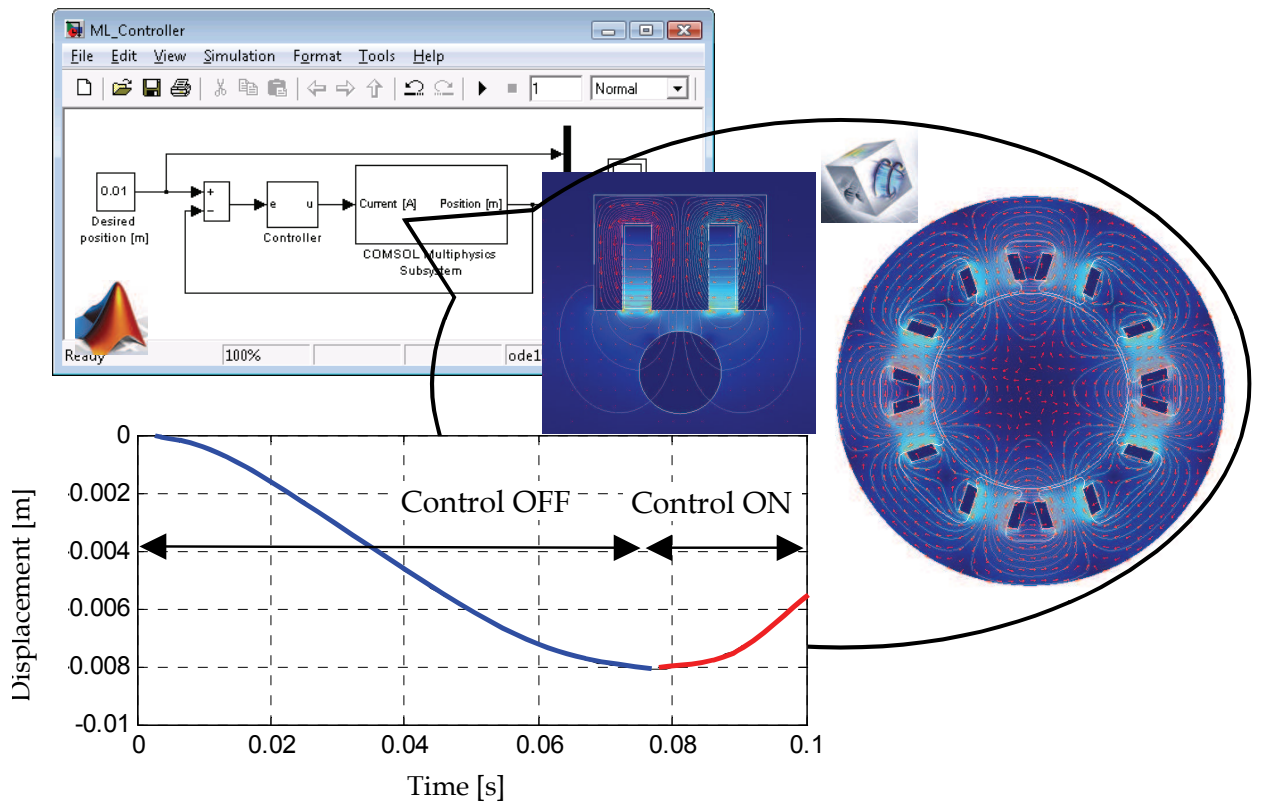

Figure 13. Interdisciplinary dynamics modelling and simulation

With this kind of modelling and simulation the power losses, eddy currents, resistive heating and more and more physical phenomena could be considered. Finally, this modelling and simulation methodology can be used in the rapid prototyping path for the real-time system operation (see Fig. 14).
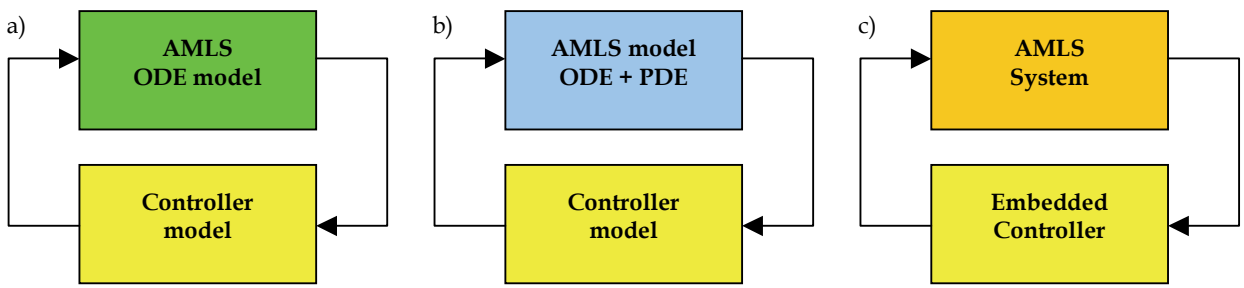

Figure 14. Model based controller design and analysis development path: a) typical, b) proposed, c) implemented

The interactive modelling and simulation are becoming an element in the controller design and development stage. The suggested controller can be validated on the realistic model of the AMLS and then successfully applied to the dedicated hardware.

\section{Conclusion}

One of the advantages of the proposed modelling and simulation methodology is the connection of many disciplines for their co-interaction to perform a multidisciplinary analysis. With the use of graphical modelling and representation of the designed 
components along with the Finite Element Method it is possible to obtain a virtual model of the designed system. Moreover, while using time-dependent solvers and simulating the prototype under the controller supervisory the system dynamics can be studied. The obtained virtual prototype contains all elements of a new device. With the detailed analysis and optimisation procedures the virtual prototype could be modified to satisfy all criteria required in the machine operation.

Nowadays, the main disadvantage of this method is the limited performance of existing computers. In this case it is impossible to simulate in the real-time the dynamics of the machine equipped with AMLS (e.g. rotor levitated in Active Magnetic Bearings). The announced development of the COMSOL Multiphysics software and application of grid computing will allow to model and simulate large-scale dynamical problems. The powerful hardware will allow to create a virtual prototype of the designed devices.

It is possible to optimise and reconfigure the AMLS construction having the geometry generated in a programmable way with respect to the required criteria.

The actual and future work will be focused on the AMLS model development to cover all possible physical phenomena, existing interactions, component properties and the controller interaction to be as close as possible to the real working system. The most important question is how to obtain such a model solvable in a real-time for control purposes (model predictive control, model reference control)? These goals could be satisfied with a wide range of identification experiments and application of the proposed methods running on a powerful computer.

Calculations based on the finite element method give a deeper insight into the phenomena of the AMLS. The performed analysis plays an important role in the design procedures of AMLS and helps in the verification of the construction assumptions. It is essential to use appropriate materials for the shaft and stator. The pole shoes arrangement, their sizes and coil parameters strongly influence the electromagnetic force value produced by the electromagnet. As shown above the design procedure of the AMLS is a complex task consisting of a few elements: analysis of the AMLS operating mode parameters, calculation of electromagnetic force, selection of materials and calculation of magnetic field properties to obtain the desired force value, and the choice of the controller architecture. The application of the COMSOL package gives a possibility to establish a connection with the MATLAB/Simulink, with data exchange. This is very useful when the multiphysic analysis is connected with the control algorithm design. The computational effort strongly depends on the mesh density.

Current author's research realized in the framework of the Polish Post-Doc project is focused on 2D and 3D modelling and analysis using the electromagnetic module together with Simulink interactions to examine the static and dynamic AMB behaviour in the real operation environment. Accordingly to the author's knowledge this kind of methodology has never been applied to Active Magnetic Levitation Systems.

\section{Acknowledgement}

Thanks to the Lord for some talents and to my family for love. Special acknowledgment goes to COMSOL A.B. (www.comsol.com) for the recent version of the COMSOL Multiphysics software and COMSOL local distributor in Poland Technika Obliczeniowa (www.tobl.com.pl). You are cordialy invited to visit the AGH page related to magnetically levitated systems www.maglev.agh.edu.pl and my private homepage 
home.agh.edu.pl/ ap. Some of presented results have been obtained during the realized research POL-POST DOC II held by Bialystok Technical University (www.air.pb.edu.pl).

\section{References}

Brauer, J. R. (2006). Magnetic Actuators and Sensors, John Wiley \& Sons, Inc., ISBN: 978-0-47173169-6, New Jersey.

Earnshaw, S. (1842). On the nature of the molecular forces which regulate the constitution of the luminferous ether., Trans. Camb. Phil. Soc., 7, pp 97-112 (1842)

Braunbeck, W. (1939). Free suspension of bodies in electric and magnetic fields, Zeitschrift für Physik, 112, 11, pp. 753-763, (1939)

Jayawant, B.V. (1981). Electromagnetic Levitation and Suspension Systems, Publishers: Edward Arnold, London, 1981

Banks, J. (ed.) (1998). Handbook of Simulation, Principles, Methodology, Advances, Applications, and Practice, John Wiley \& Sons, Inc., ISBN 0-471-13403-1, New York

Comsol 3.3 (2007). User's Guide and Introduction, COMSOL AB, Sweden.

Gosiewski, Z. (1993). Magnetic bearings for rotary machinery. Theoretical Basis.(in Polish) Wyższa Szkoła Inżynierska, Koszalin 1993.

Gosiewski, Z. \& Falkowski, K. (2003), Multifunction magnetic bearings, (in Polish), Biblioteka Naukowa Instytutu Lotnictwa. Warszawa,

Grega, W. \& Piłat, A. (2001). A Comparison of Nonlinear Controllers for Magnetic Levitation System. Proc. of $5^{\text {th }}$ World Multiconference on Systemics, Cybernetics and Informatics, July 2001, Orlando-Florida, USA

Habermann, H. \& Liard, G. (1979). Practical Magnetic Bearings, IEEE Spectrum, Vol. 16, No. 9, September, 1979

Hammond, P. \& Sykulski J. K. (1994). Engineering Electromagnetism - Physical Processes and Computation, Oxford University Press, ISBN 0-19-856289-6, New York.

Kanebako, H. \& Okada, Y. (2002), New Design of Hybrid type Self-Bearing Motor for HighSpeed Miniature spindle, Proc. of The Eighth International Symposium on Magnetic Bearings, August 2002, Mito, Japan

Kozanecka, D. (2000). Digitaly controlled magnetic bearing. (in Polish). Zeszyty Naukowe Politechniki Łodzkiej, Łódź, 2000.

Maslen, E. (1995). Magnetic Bearings. University of Virginia, Department of Mechanical, Aerospace and Nuclear Engineering, Charlottesville, Virginia, 1995.

Meeker, D.C \& Maslen, E. (2006). Analysis and Control of a Three Pole Radial Magnetic Bearing, Proceedings of $10^{\text {th }}$ International Symposium on Active Magnetic Bearings, August 2006, Martigny, Switzerland

Noh, M. D. \& Maslen, E. H.: Self Sensing Active Magnetic Bearings Based on Parameter Estimation. IEEE Transactions on Instrumentation and Measurement, 46(1), 1997 February, pp. $45 \div 50$.

Ohmori, K.; Kim, S.; Masuzawa, T. \& Okada, Y. (2002). Design of an Axial-type Self Bearing Motor for Small Axial Pump, Proc. of The Eighth International Symposium on Magnetic Bearings, pp. $15 \div 20$, August 2002, Mito, Japan

Piłat, A. (1999a). Magnetic levitation - system description. AGH, Kraków 1999 (in Polish)

Piłat, A. (1999b). Genetic algorithms applied to optimal PID controller tuning for magnetic levitation system. Proc. of Computer Methods and Systems, pp. $271 \div 276$, October 1999, Kraków, Poland. 
Piłat, A. (2000). Feedback Linearization and LQ Control for Magnetic Levitation System. $6^{\text {th }}$ International Conference on Methods and Models in Automation and Robotics, pp. $407 \div$ 412, August 2000, Międzyzdroje, Poland,.

Piłat, A. (2001) Time-Optimal Control for Magnetic Levitation System. $7^{\text {th }}$ International Conference on Methods and Models in Automation and Robotics, pp. 873-878, August 2001, Międzyzdroje, Poland,

Piłat, A. (2002a). Control of magnetically levitated systems. Ph.D. Dissertation (in Polish), AGH University of Science and Technology, Kraków, Poland

Piłat, A. (2002b). Feedback Linearization Control of AMB System. Proceedings of The Eight International Symposium on Magnetic Bearings, August 2002, Mito, Japan

Piłat, A. (2003). Fuzzy logic controller for Active Magnetic Bearing. Proceedings of Active Noise and Vibration Control Methods, May 2003, Kraków, Poland

Piłat, A. (2004). FEMLab software applied to active magnetic bearing analysis, International Journal of Applied Mathematics and Computer Science. 2004, vol. 14 no. 4 spec. iss.: Issues in modelling, optimization and control pp. $497 \div 501$

Piłat, A. (2005a). Programmable analog hardware for control systems exampled by magnetic suspension, Proc. of Computer Methods and Systems, pp.143-148, November 2005, Kraków, Poland

Piłat, A. \& Turnau A. (2005b). Self-organizing fuzzy controller for magnetic levitation system, Proc. of Computer Methods and Systems, pp. 101-106, November 2005, Kraków, Poland

Piłat, A. \& Grega W. (2005c). Reconfigurable test-rig for AMB control, Proceedings of $7^{\text {th }}$ Conference on Active Noise and Vibration Control Methods, pp. 1-8, June 2005, Wigry, Poland

Piłat, A. (2006). PD control strategy for 3 coils AMB, Proceedings of 10th International Symposium on Active Magnetic Bearings, August 2006, Martigny, Switzerland

Piłat, A. (2007). Automatic generation of Active Magnetic Bearing geometry with COMSOL Multiphysics, Proc. of Computer Methods and Systems CMS'07, pp. 147-150, Krakow, November 2007, Krakow, Poland

Rothwell, E. J. \& Cloud, M. J. (2001). Electromagnetics, ISBN 0-8493-1397-X, CRC Press, Boca Raton.

Sinha, P. K. (1984). Design of Magnetically Levitated Vehicle. IEEE Transactions On Magnetics, vol. MAG-20, no. 5, September 1984, pp. $1672 \div 1674$.

Sinha, P. K. (1987). Electromagnetic Suspension. Dynamics \& Control. Peter Perginus Ltd., London.

Silva, Clarence de W., (2000), Vibration Fundamentals and Practice, CRC Press 2000.

Schweitzer, G. (1976). Characteristics of a Magnetic Rotor Bearing for Active Vibration Control, Paper C239/76, Proc. of First International Conference on Vibrations in Rotating Machinery, (1976)

Schweitzer, G.; Traxler, A. \& Bleuler, H. (1994). Magnetlager. Springer-Verlag.

Yeadon, W. H. (ed.) \& Yeadon A.W., (2001). Handbook of small electric motors, McGraw-Hill, ISBN 0-07-072332-X, New York.

Zienkiewicz, O. C. \& Taylor, R. L. (2000). The Finite Element Method. Vol. 1. The Basis. $5^{\text {th }}$ ed., Butterworth-Heinemann, ISBN 075065049 4, Oxford 


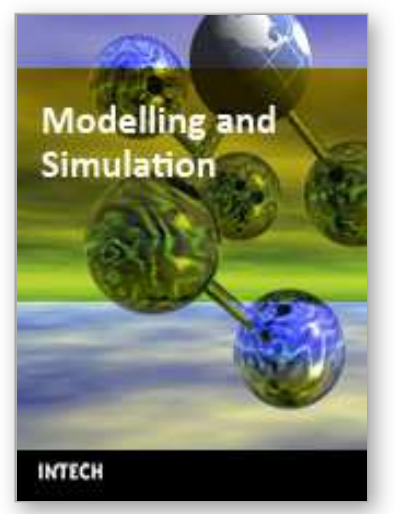

\author{
Modelling and Simulation \\ Edited by Giuseppe Petrone and Giuliano Cammarata
}

ISBN 978-3-902613-25-7

Hard cover, 688 pages

Publisher I-Tech Education and Publishing

Published online 01, June, 2008

Published in print edition June, 2008

This book collects original and innovative research studies concerning modeling and simulation of physical systems in a very wide range of applications, encompassing micro-electro-mechanical systems, measurement instrumentations, catalytic reactors, biomechanical applications, biological and chemical sensors, magnetosensitive materials, silicon photonic devices, electronic devices, optical fibers, electro-microfluidic systems, composite materials, fuel cells, indoor air-conditioning systems, active magnetic levitation systems and more. Some of the most recent numerical techniques, as well as some of the software among the most accurate and sophisticated in treating complex systems, are applied in order to exhaustively contribute in knowledge advances.

\title{
How to reference
}

In order to correctly reference this scholarly work, feel free to copy and paste the following:

Adam Krzysztof Pilat (2008). Active Magnetic Suspension and Bearing, Modelling and Simulation, Giuseppe Petrone and Giuliano Cammarata (Ed.), ISBN: 978-3-902613-25-7, InTech, Available from:

http://www.intechopen.com/books/modelling_and_simulation/active_magnetic_suspension_and_bearing

\section{INTECH}

open science | open minds

\section{InTech Europe}

University Campus STeP Ri

Slavka Krautzeka 83/A

51000 Rijeka, Croatia

Phone: +385 (51) 770447

Fax: +385 (51) 686166

www.intechopen.com

\section{InTech China}

Unit 405, Office Block, Hotel Equatorial Shanghai

No.65, Yan An Road (West), Shanghai, 200040, China

中国上海市延安西路65号上海国际贵都大饭店办公楼 405 单元

Phone: +86-21-62489820

Fax: $+86-21-62489821$ 
(C) 2008 The Author(s). Licensee IntechOpen. This chapter is distributed under the terms of the Creative Commons Attribution-NonCommercialShareAlike-3.0 License, which permits use, distribution and reproduction for non-commercial purposes, provided the original is properly cited and derivative works building on this content are distributed under the same license. 